\title{
Robust Speckle Detection in Ultrasound Images. Evaluation Aspects
}

\author{
R.Martí $^{a}$ and J.Martí ${ }^{a}$ and J.Freixenet ${ }^{a}$ and J.C.Vilanova ${ }^{b}$ and J.Barceló ${ }^{b}$ \\ ${ }^{a}$ Computer Vision and Robotics Group - University of Girona, Spain \\ ${ }^{b}$ Girona Magnetic Resonance Imaging Center, Spain
}

\begin{abstract}
The characterisation and posterior detection of speckle noise in ultrasound (US) has been regarded as an important research topic in US imaging, mainly focusing on two specific applications: improving signal to noise ratio by removing speckle noise distribution and, secondly, detecting fully developed speckle patterns in order to perform a 3D reconstruction using only image content information from freehand sensorless images.

The main novelty of this work is to show that speckle detection can be improved based on finding optimally discriminant low order speckle statistics. We describe a fully automatic method for speckle detection and propose and validate a framework to be efficiently applied to real B-scan data, not being published to date. Different experiments have been carried out in order to validate the speckle detection methodology using both real and simulated data.
\end{abstract}

Keywords: Ultrasound, Speckle detection, Fractional low order moments, Discriminant analysis

\section{BACKGROUND AND MOTIVATION}

The basis of US imaging is based on capturing how different tissues scatter or reflect the sonic pulses. Reflection and scattering are closely related concepts, basically the difference lies on the particle size of this interaction. Sound is scattered or reflected mainly due to non-homogeneity or different compressibility areas found in the imaged tissue. Taking into account an spatially randomly distributed population of sub-resolution scatterers, one can talk about incoherent scattering which gives rise to speckle noise or fully developed speckle. If this distribution follows a given pattern, a coherent component is introduced. Fully developed speckle has been shown to follow a Rayleigh point distribution function $(P D F)$, whereas as the coherent component increases the signal behaves as a Rician PDF characterised by the parameter $k$, the ratio between coherent and diffuse signal.

In terms of simulating speckle patterns, speckle can be modeled as a sum of backscatter signals emitted from a given number of points in the imaged area. This summation can be expressed using phasor notation as,

$$
A=s+\sum_{i=1}^{\mu} a_{i}
$$

where $A$ is a single amplitude sample (which is related to the grey-level intensity of a B-scan). The parameter $s$ represents the coherent signal and $a_{i}$ the random (also referred to as diffuse) component. A single sample $A$ can be simulated following the above equation: a single vector $s$ with zero phase and amplitude $k$ (the coherent signal) is added to the sum of $\mu$ vectors of length $\sqrt{2 / \mu}$ and random phase which conforms the diffuse component. ${ }^{1}$ In order to increase image contrast, usually the final grey-level intensity of a B-scan is given by a logarithmic compression of the original amplitude signal. Hence, to be able to obtain a good characterisation of the speckle in Ultrasound images, B-scan information needs to be decompressed. Several authors suggest a mapping of the form $I=D \ln (A)+G$, where $I$ is the final B-scan intensity, $D$ a compression factor, $G$ an offset value and $A$ the original amplitude signal. The offset value is often disregarded as it does not affect the statistics of the speckle, hence being $D$ the important factor to be determined in order to obtain a good speckle detection.

Corresponding author Robert Martí. Edifici P-IV, Av. Lluís Santaló, s/n, 17071. Girona, Spain. E-mail: marly@eia.udg.es 
The main aim of this work is to provide an automatic method for the detection of fully developed speckle patterns in B-scan images. One common approach to this detection is to describe speckle using a known statistical model. Various models have been proposed for speckle characterisation, Rayleigh and Rician models were originally proposed but more general models such as the Nakagami, ${ }^{2}$ Generalised Gamma $\left({ }^{3}\right), K,{ }^{4}$ Generalised $K$ and Homodyned $K$ distributions ${ }^{5-7}$ have been shown to account for better speckle description at the expense of a more complex formulation. The aim of those methods is to obtain an initial estimate the model parameters based on extracting statistical features from the experimental data, commonly moments of different orders. Using those features a Maximum Likelihood Estimation $(M L E)$ or Maximum a Posteriori $(M A P)$ approach is applied to obtain the final model parameters.

Instead of using a specific statistical distribution, an alternative approach, which is adopted here, is to describe speckle based on statistical features directly computed from the amplitude distribution. These features are typically extracted from the amplitude moments of the US image (or B-scan). A classification model, typically based on clustering techniques ${ }^{8,9}$ or application-specific discriminant functions ${ }^{10}$ is built on trained speckle and non-speckle data, and is subsequently used for classifying new features as being speckle or non-speckle. In order words, speckle detection becomes a two-class pattern recognition problem.

In any case, an additional important issue which needs to be addressed is that the final intensity of a B-scan is usually log-compressed by the ultrasound machine. Hence, a prior decompression of the intensity signal needs to be estimated. This work describes a speckle detection methodology based on extracting optimally discriminant low order speckle statistics and simultaneously estimating the decompression factor of the data.

The paper is structured as follows Sect. 2 proposes a fully automatic approach for speckle detection in B-scan data and Sect. 3 shows real and simulated results. The paper finishes with some conclusions and future work.

\section{MATERIALS AND METHODS}

As previously stated, our work builds up on the early speckle detection methodology proposed by Prager et al., ${ }^{1}$ but incorporates novel aspects such as the optimal selection of the power statistics applied to real B-scans and removes the need of manual intervention, aspects which we believe make the method more robust.

\subsection{Speckle Characterisation}

Speckle in ultrasound images is commonly characterised by using three parameters: the coherent signal energy $s^{2}$, the diffuse signal energy $2 * \sigma^{2}$ and the number of scatters per resolution cell $\mu$. The coherent and diffuse signals are also commonly expressed as the ratio $k=s / \sigma$, the proportion of coherent to diffuse signal. As demonstrated by different authors, ${ }^{11,12}$ speckle can be characterised by two low order moments: the ratio between the mean and the standard deviation $(R)$ and the skewness $(S)$, both are defined as follows,

$$
R=\frac{E\left\{A^{v}\right\}}{\sqrt{E\left\{A^{2 v}\right\}-E^{2}\left\{A^{v}\right\}}} \quad S=\frac{E\left\{\left(A^{v}-E\left\{A^{v}\right\}\right)^{3}\right\}}{\left(E\left\{A^{2 v}\right\}-E^{2}\left\{A^{v}\right\}\right)^{3 / 2}}
$$

where A is the signal amplitude, and $v$ the power of the statistical moment. Effectively, $R$ and $S$ can be computed using $v$ values different from one. This issue is important as the use of an specific value of $v$ could lead to a better discrimination between speckle and non-speckle signals. For instance, in all experiments described by Prager et al. ${ }^{1}$ a value of $v=1.8$ was used. This value was qualitatively obtained using simulated data. As noted in, ${ }^{8}$ this assertion may not be always valid, they showed that an analysis of the discriminant power of the $R$ - $S$ features should be carried out in order to determine the optimal order of the statistics.

\subsection{Speckle Detection}

Prager et al. proposal is based on a simultaneous method for estimating B-scan decompression parameters and subsequently detect speckle regions based on the ellipsoid discriminant function obtained from the $R-S$ statistical features from the data. For the sake of completeness their algorithm is described below.

1. Obtain an ellipse discriminant function using simulated speckle data. 
2. Choose an initial compression value $D$.

3. Manually label speckle patches in the real B-scan images (in their experiments 40 patches are used).

4. For each patch

(a) Decompress the patch intensity using $I=\exp (p / D)$.

(b) Compute $R-S$ features from $I$, using a $v$ value arbitrarily set to 1 .

(c) Using the ellipse discriminant function, compute the feature error values. If the error is large, use an optimisation algorithm in order to minimise the error w.r.t. $D$, obtaining the estimation of the decompression factor $(D)$.

5. Adapt the ellipse center parameters using the mean $R-S$ features from the manually labeled patches.

6. For all patches in the image, decompress it using the $D$ value, obtain $R-S$ features and use the ellipse discriminant function to assert if it is a speckle patch (is inside the ellipse).

The above approach presents some drawbacks. A first problem is the need of manually detecting initial speckle regions in order to extract sample statistics. Manual intervention is also needed for the definition of the discriminant ellipse parameters. Another important drawback is the fact that $R-S$ are computed using an arbitrarily order ( $v=1.8$ for simulated data and $v=1$ for real B-scans). Our proposal tackles the above problems, proposing a novel method for automatically detecting initial speckle patches (referred to here as core patches) and selecting the most discriminant features for speckle detection. The method is described by the following steps,

1. Obtain an ellipse discriminant function from speckle simulated data for different $v$ values ranging from 0 to 3 .

2. Automatically detect core speckle and non-speckle regions and decompression factor $D$ from real B-scan data (see next subsection).

3. Using speckle and non-speckle, compute $R-S$ statistics and find $v_{o p t}$, the $v$ value where those statistics are optimally discriminant.

4. Modify the parameters of the ellipse discriminant function, as in step 5 of the original method.

5. For all patches in the image, decompress and test if it is speckle, as in step 6 of the original method.

Authors are aware that different key aspects of their method need to be thoroughly described and evaluated, namely steps 2 and 3, core speckle detection and the optimally discriminant statistics, both are described in the sections below.

\subsection{Core Speckle and Non-Speckle}

The idea behind the detection of speckle and non-speckle patches is based on the assumption that the decompression values $D$ found after optimisation (step $4 \mathrm{c}$ of the original work) are stable as a function of $v$ for speckle patches. For the case of non-speckle patches, $D$ values will present high variability as a function of $v$, explained by the fact that optimisation will be unable to find a meaningful $D$ value. The proposed core speckle detection works as follows: for a test B-scan image a number of randomly distributed samples are obtained. For each sample a $D$ value is estimated using an optimisation algorithm (i.e. Levenberg-Marquardt), similar to step 4c of the original work. For a speckle pattern, the estimated $D$ value is likely to be stable as a function of $v$, whereas for a non-speckle patch, the $D$ value will vary providing unreliable estimations. This assumption has been corroborated through different simulated and empirical experiments, some of them shown in the evaluation section. The final estimate of the compression factor will be obtained analysing the most stable values (i.e. the median value computed from values with the smallest fluctuation). In addition, the patches closest to this estimation will be regarded as the core speckle patches. The reader is referred to author's related work ${ }^{13}$ where the speckle detection is discussed in more detail. 


\subsection{Discriminant Analysis}

Other authors have already justified the need of finding optimal discriminant statistics for speckle detection (see ${ }^{8}$ ). However, the authors applied the discriminant analysis only to speckle simulated data, not to real B-scan images. Nevertheless, this issue needs to be investigated as a different speckle detection approach is adopted here and moreover, the discriminant analysis applied to real B-scan data conforms one of the novel aspects of our approach.

The $R-S$ statistics can be regarded as features for a classic pattern recognition problem: ${ }^{14}$ given a set of feature values classify them as being speckle or non-speckle. As a set of $R$ - $S$ features is obtained for each sampled $v$ value, one could think that the most appropriate features are those which maximise a certain measure of discriminating power. One of the most commonly used methods is the analysis of the within class $\left(S_{w}\right)$ and the between class $\left(S_{b}\right)$ scatter matrices. ${ }^{14}$ The within class scatter matrix, $S_{w}$, assuming equally probable classes is defined as,

$$
S_{w}=\frac{1}{c} \sum_{i=1}^{c} E\left(X-m_{i}\right)\left(X-m_{i}\right)^{t}
$$

where $c$ is the total number of classes, $X$ is the feature vector (i.e. $N$ samples of $R$ - $S$ values) and $m_{i}$ is the mean feature vector for class $i$. The between class scatter matrix, $S_{b}$, is related to the mean differences of each class compared to the total mean for all classes $(M)$, and is defined as,

$$
S_{b}=\frac{1}{c} \sum_{i=1}^{c} E\left(m_{i}-M\right)\left(m_{i}-M\right)^{t}
$$

Defining the matrix $S_{m}$ as the sum of the $S_{w}$ and $S_{b}$ scatter matrices, different measures of discrimination power can be computed. In order to follow a consistent notation with, ${ }^{8}$ those measures are referred to as $J_{1}, J_{2}$ and $J_{3}$ and are defined as follows.

$$
J_{1}=\operatorname{trace}\left(S_{m}\right) / \operatorname{trace}\left(S_{w}\right) \quad J_{2}=\operatorname{det}\left(S_{m}\right) / \operatorname{det}\left(S_{w}\right) \quad J_{3}=\operatorname{trace}\left(S_{w}^{-1} * S_{b}\right)
$$

For all cases a higher value denotes higher class separability, although this criteria does not always coincide for all measures, which, excerpted from the experiments, is specially true for $J_{1}$ measures. Back to the problem of speckle detection, having those measures of class separability, $R-S$ features used for speckle detection will be the ones computed using the value of $v$ which maximises class separability (referred to as the $v_{o p t}$ value).

\section{EVALUATION AND RESULTS}

\subsection{Simulated Data}

As an initial evaluation, different experiments have been carried out in order to validate the speckle detection framework using simulated data. Simulated speckle data has been generated using different $k$ and $\mu$ parameters. From a total of 8 different sets of 4000 samples simulating speckle and non-speckle data, $R-S$ features have been computed as a function of $v$. Figure 1(b) shows a scatter plot of these features labeled as being speckle $(\cdot)$ and non-speckle $(+)$. Figure 1(a) shows the discriminant analysis using the $J_{i}$ measures for this data. Clearly, both $J_{2}$ and $J_{3}$ coincide to a optimal $v$ value $\left(v_{\text {opt }}\right)$ at 1.1 . The scatter plot in Fig. $1(\mathrm{~b})$ is obtained using this $v_{\text {opt }}$ value. Fully developed speckle data, although simulated using four different parameters is nicely clustered, while some of the non-speckle data overlaps with the speckle mainly due to its similar parameters. This figure also shows the ellipse discriminant function, automatically obtained from the speckle data, which provides a robust and computationally efficient method for speckle detection. Using this ellipsoid function, the figure also shows detection results, where grey samples depict detection errors (grey dots are false negatives and grey crosses are false positives). This experiment provides information about the usefulness of the ellipsoid function and the suitability of performing the discriminant analysis. For the $v_{o p t}$ value, we obtain $91 \%$ of correct classification, with a lower accuracy for the other $v$ values, which suggest that the use of optimal discriminant analysis has a positive impact in the detection of speckle. 


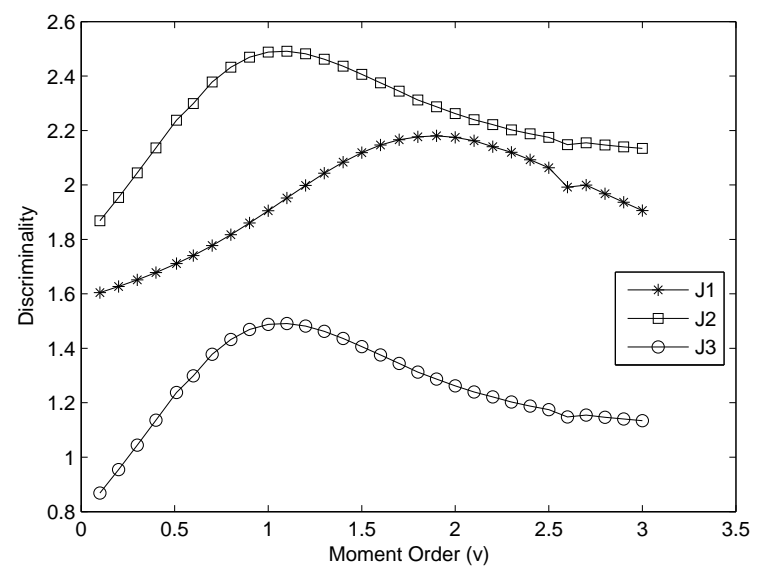

(a)

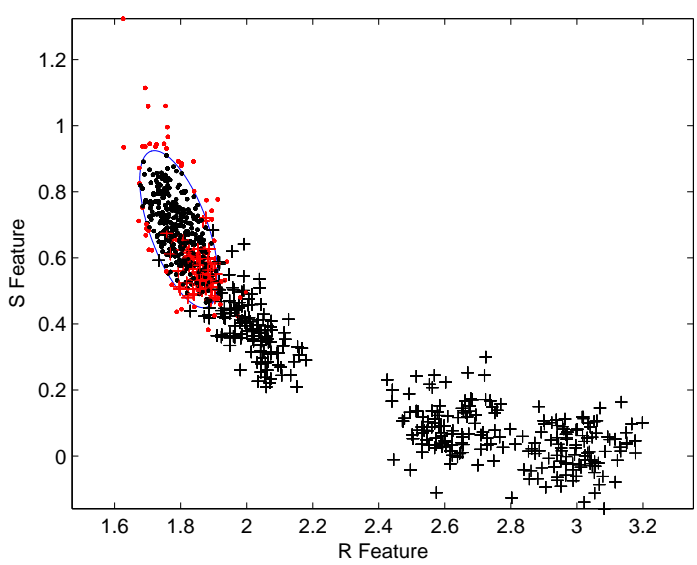

(b)

Figure 1. Simulated results. (a) Data discrimination as a function of the $v$ value ( $x$ axis) and (b) $R-S$ scatter plot at $v_{\text {opt }}$ (see main text for explanation).

\subsection{Core Speckle Detection}

Evaluation of the core speckle and non-speckle detection algorithm described above is presented in this section. Different simulated and experimental results have been performed in order to empirically show the validity of the assumption that speckle patches provide an stable decompression estimation compared to non-speckle patches. As an initial experiment, Fig. 2 shows the variability of the $D$ estimation as a function of $v$ for speckle (dotted) and non-speckle (solid) patches manually defined in a real B-scan image. It can bee seen from those results that speckle patches present a low variability compared to non-speckle samples. Other experiments (results not shown here) using simulated data (similar to the one described in the previous section) and different real B-scan images obtained similar trends.

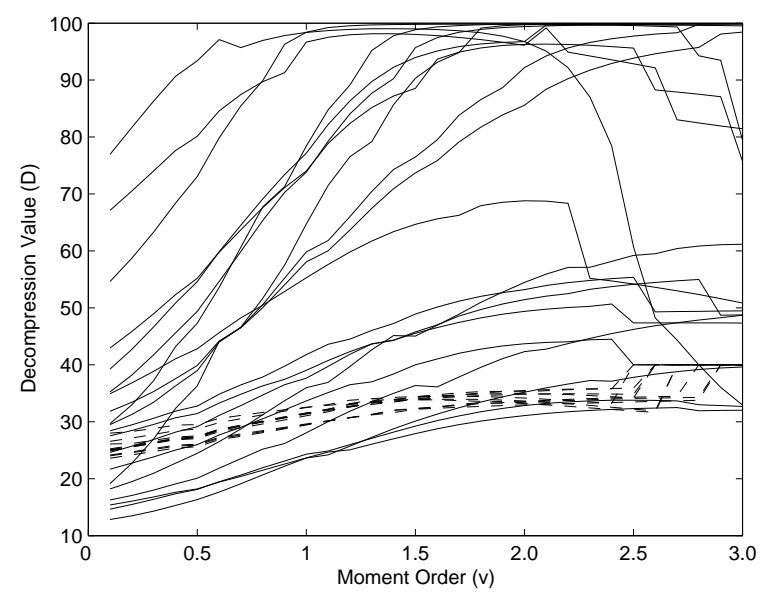

Figure 2. Core speckle and non-speckle detection: $D$ estimation as a function of $v$ using manually labeled speckle (dotted) and non-speckle (solid) patches.

Automatically extracting the most stable estimations of the decompression value will provide the core speckle patches, whereas the most unstable results will be defined as the non-speckle patches. Both speckle and nonspeckle patches will be used for obtaining the $R-S$ features used for computing the optimally discriminant 
$v_{\text {opt }}$ value. Figure 3 shows core speckle and non-speckle detection results in real B-scan data for prostatic phantom and liver images. In both cases, core speckle clearly shows typical low intensity fully developed speckle patches, whereas core non-speckle are characterised by the high contrast regions with important coherent signal components.

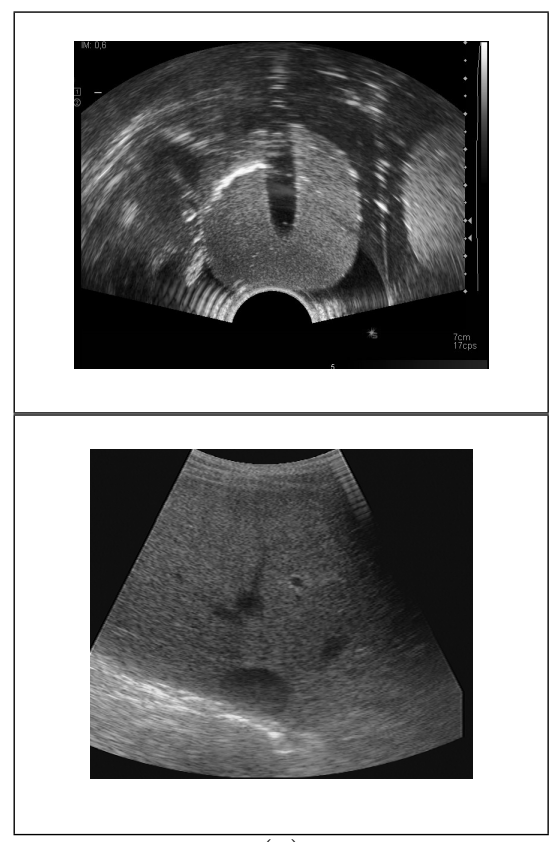

(a)

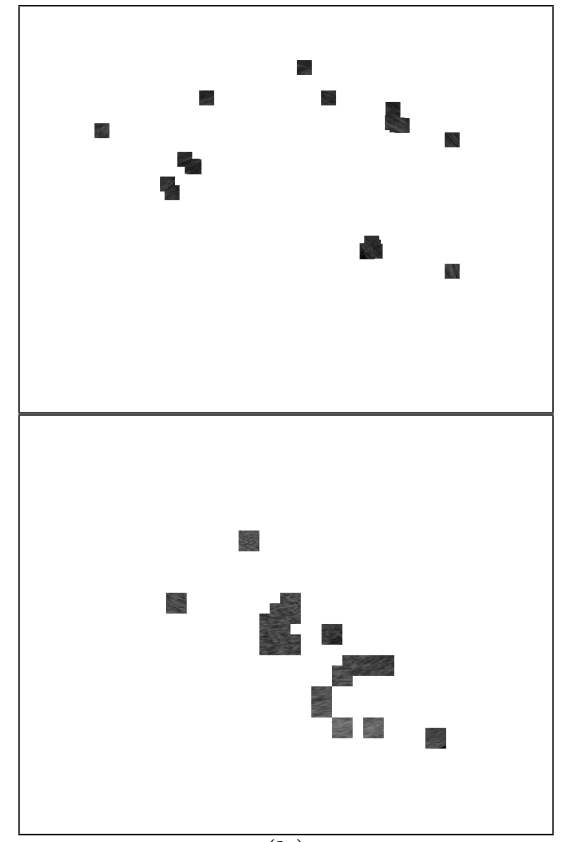

(b)

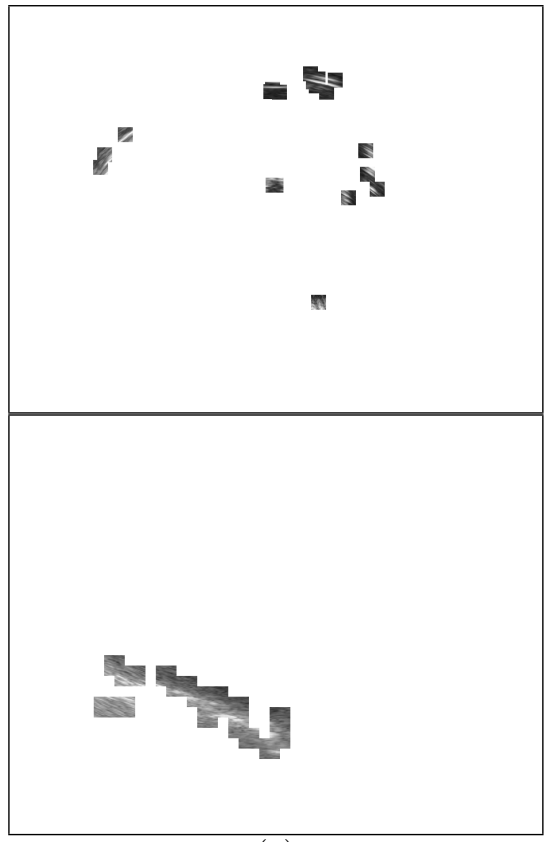

(c)

Figure 3. Results for core speckle detection in prostatic phantom (top) and liver (bottom) images. (a) original B-scan, (b) core speckle, (c) core non-speckle patches.

In addition to the log compressed image, some modern ultrasound machines provide the uncompressed echo amplitude signal. In that case, the method would not need to estimate the decrompression parameter, making the core speckle and non-speckle step less computationally costly, avoiding the use of the optimisation algorithm in order to obtain the estimation of the decompression factor $D$. However, if this information is unavailable, for instance due to the limitations of the ultrasound scanner or to the fact that images are from retrospective studies (where non-compressed images are not available), the presented method provides an estimation of this compression.

\subsection{B-scan Images}

The proposed algorithm has been tested using prostatic and liver US images. Although a single image was used for the core speckle detection for each data set, (the ones shown in Fig. 3), detection results are qualitatively satisfactory as shown in Fig. 4 and Fig. 5. For the prostatic phantom (Fig. 4), two different non-consecutive scans have been tested. In both B-scans the method interestingly detects regions not only with dark speckle patches (similar to the core speckle) but also lighter speckle areas inside the prostate area.

Figure 5 shows speckle detection results for two different B-scans of a liver. In this case, images show less obvious structured patterns compared to prostatic phantom images. The method successfully detects a large number of speckle patterns avoiding the small darker structures (i.e. vessels) and the brightest areas with larger coherent components.

Similarly to the simulated case, the discriminant analysis of the phantom results of Fig. 4 is shown in top row of Fig. 6(a). In this case, although this is not simulated data, a clear maximum is observed, specially for the $J_{2}$ and $J_{3}$ measures at $v_{\text {opt }}=1.8$. This value was the value used for obtaining the results previously shown in Fig. 4. The $v_{\text {opt }}$ value coincides with the qualitative analysis of both $R$ and $S$ features shown in top row of 


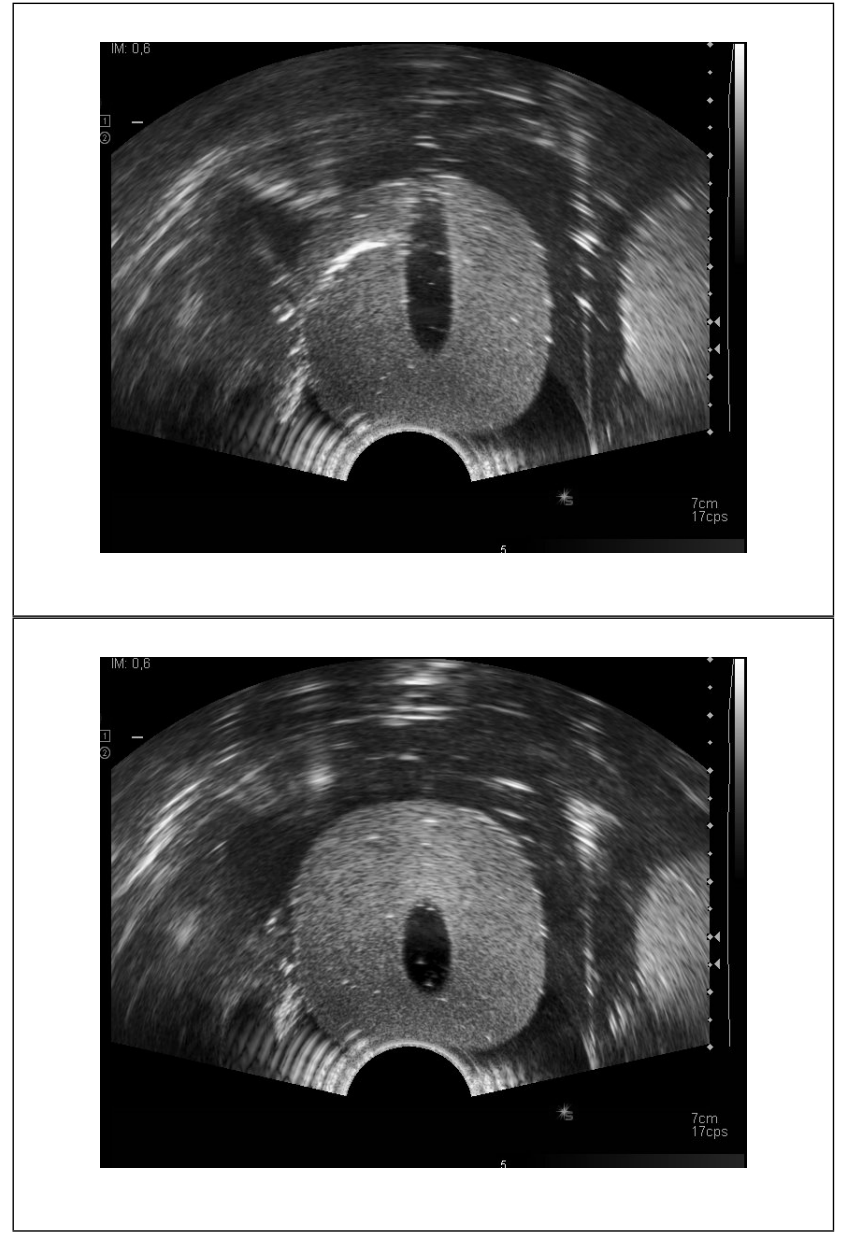

(a)
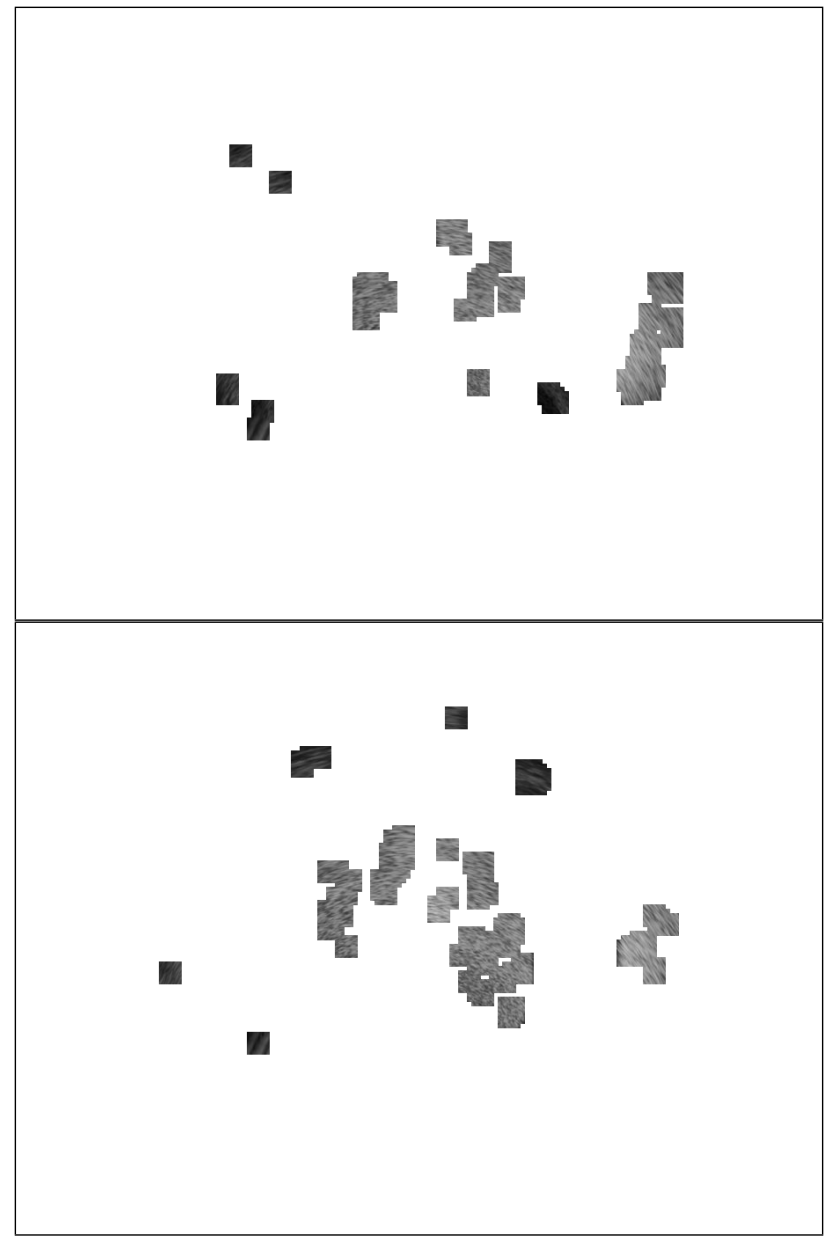

(b)

Figure 4. Speckle detection results for prostatic phantom images. (a) original and (b) speckle detection.

Fig. 6(b),(c), where a better discrimination can be appreciated around the 1.8 value, specially clearer for the $R$ features. Bottom row of Fig. 6 shows discriminant analysis for speckle detection for liver images shown in Fig. 5. For this particular case, the $v_{o p t}=0.3$, a value different from the phantom case, illustrating the need of the discriminant analysis step for an optimal speckle detection. As in the prostatic phantom case, the $R$ and $S$ features are also shown, where a less clear but distinguishable speckle and non-speckle discrimination can be appreciated around $v_{\text {opt }}$.

\subsection{Computational Cost}

Although the implementation of the proposed method has not been computationally optimised, it could be interesting to provide an indication of its computational cost, specially thinking of using the proposed methodology in clinical applications. The proposed method consists of two different steps: building the discriminant function based on simulated and real train data (steps 1 to 4 of the proposed method) and the detection of speckle patterns in test images (step 5). The former, model building, takes must of the computation time, mainly due to the large number of randomly sampled patches used for core speckle and non-speckle detection step. However, one should note that the computation of a new model is needed only when the ultrasound imaging parameters or the imaged tissues change. Once the model is built, for the second step, the detection of speckle patches given a set of B-scan images, the computation is quite efficient, only needing to extract the $R-S$ features for each patch in the image and test if it lies within the ellipse discriminant function. In this work, the model building was implemented using MATLAB (The Mathworks Inc, Natick, MA) while for the speckle detection $\mathrm{C}++$ code 


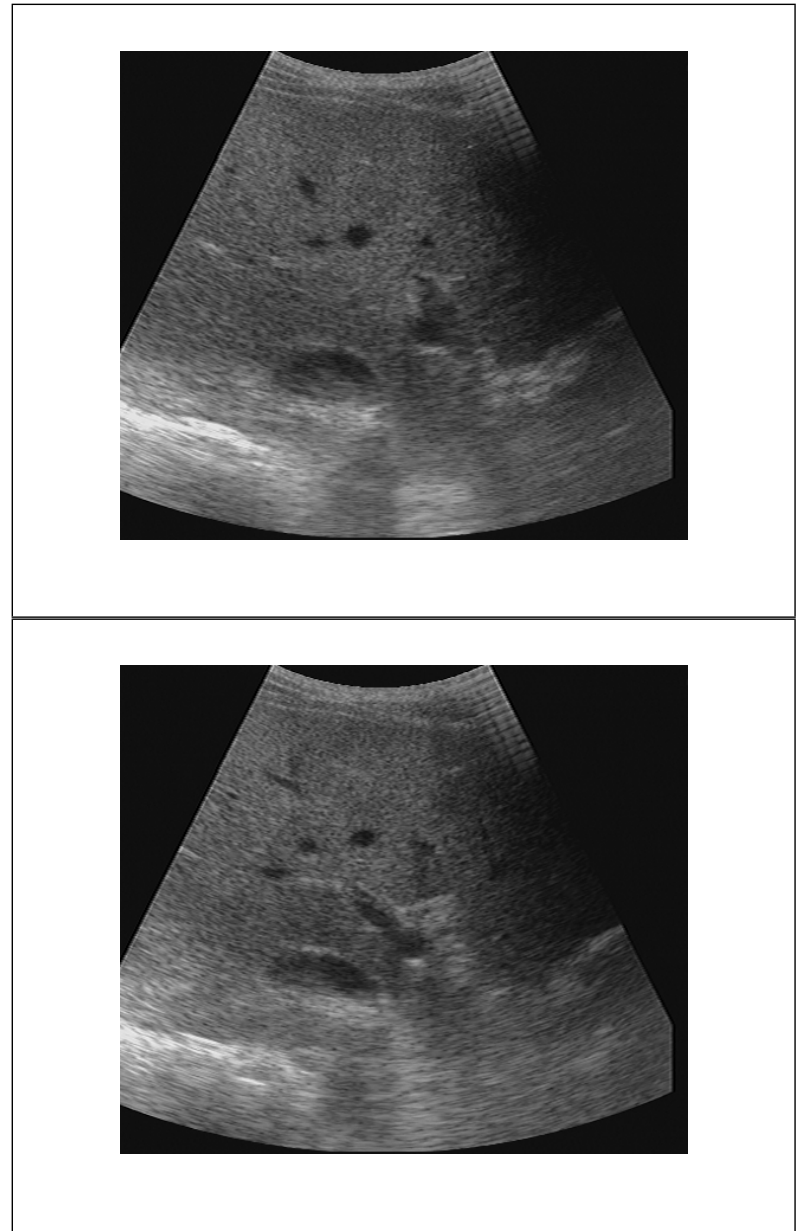

(a)

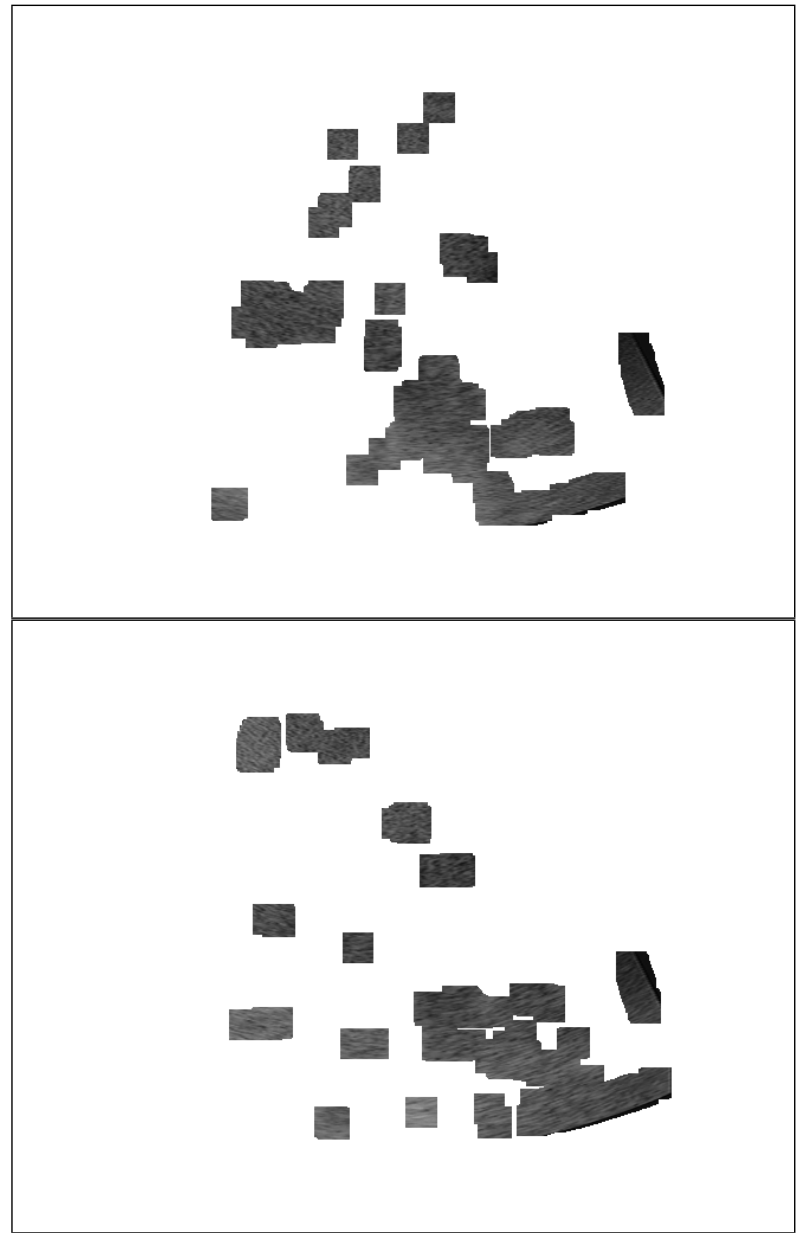

(b)

Figure 5. Speckle detection results for liver images. (a) original and (b) speckle detection.

was used. As an indication and keeping in mind a non-optimised implementation, the model building takes approximately 5 minutes depending on the image size (computation time could be greatly improved using other programming platforms) and around 2 seconds for the detection of speckle patches in a B-scan image of approximately 700 by 700 pixels. In addition, for the case when uncompressed B-scan images are available directly from the ultrasound machine, the model building step will be much more computationally efficient, removing the need of using the non linear optimisation method for the estimation of the decompression parameter, as described in the core speckle section.

\section{DISCUSSION AND CONCLUSIONS}

A methodology for the detection of speckle patterns in B-scan ultrasound images has been presented. The proposed method stems from a previously published proposal based on the computation of low order statistics from the raw envelope signal. Nevertheless, important novel aspects are introduced such as optimally discriminant features and the suppression of the need of manual intervention. Various quantitative and qualitative evaluation results using both simulated and real data have been provided which show the effectiveness of our approach. In particular, it has been shown the need for using discriminant analysis in order to determine the optimal power of the statistical moments and that this optimal value strongly depends on the characteristics and imaged tissues found in the B-scan data. This has been made specially clear as different optimal values have been obtained for simulated data and prostatic phantom and liver images. The detected speckle patches obtained with the proposed 

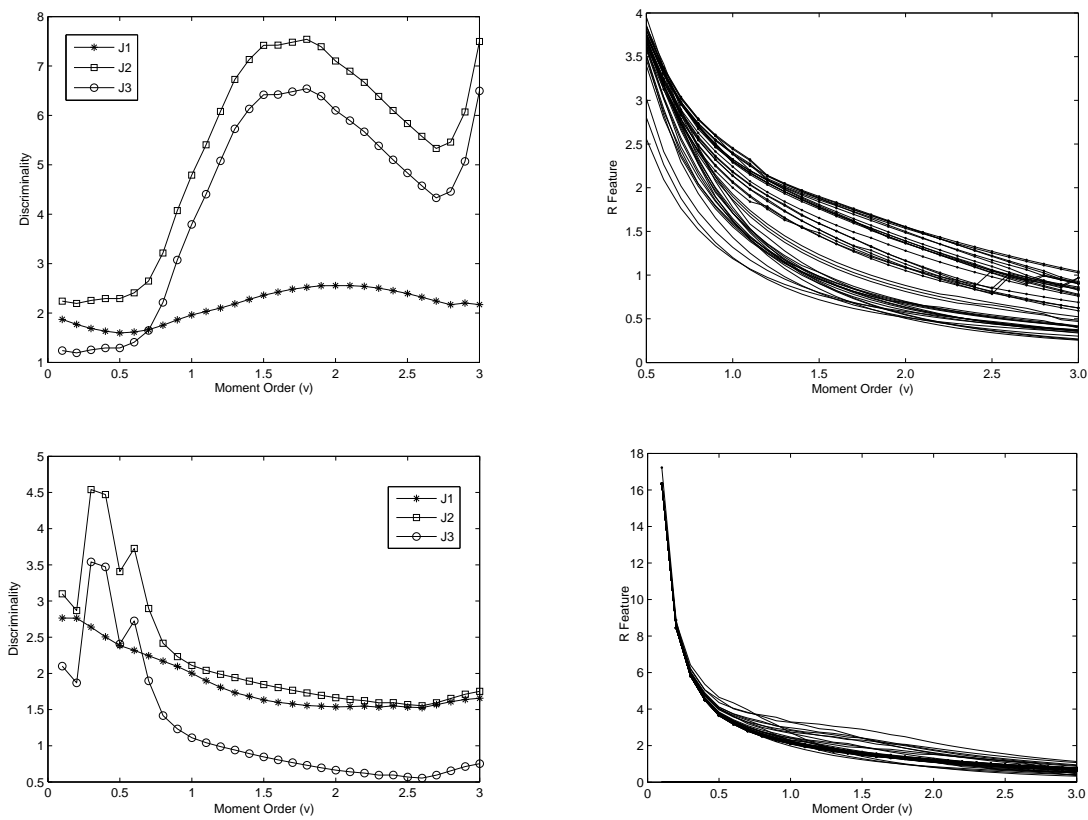

(a)

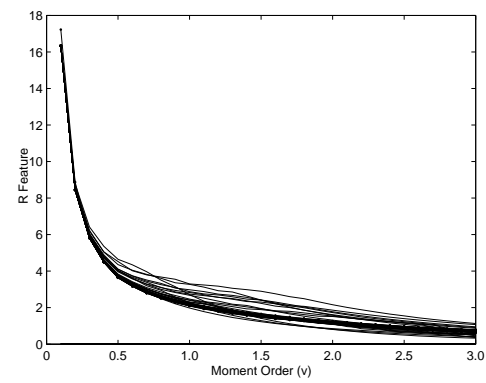

(b)
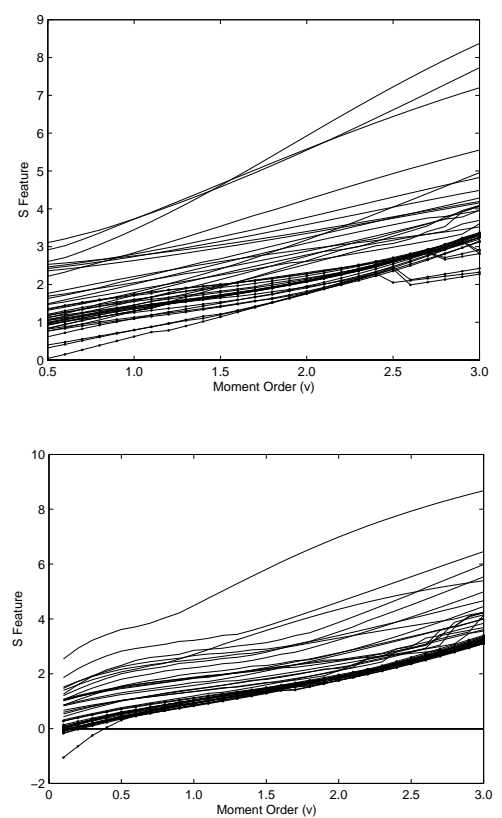

(c)

Figure 6. (a) Discriminant analysis as a function of $v$. Statistical (b) $\mathrm{R}$ and (c) $\mathrm{S}$ features as a function of $v$ are also shown. Top and bottom rows refer to phantom prostatic images and to liver images, respectively.

method can be used in other algorithms in terms of adaptive speckle suppression, ${ }^{15}$ and speckle de-correlation approaches for sensorless freehand 3D reconstruction ${ }^{16}$ or elastography ${ }^{17}$ using ultrasound image sequences.

Future work will incorporate a large scale evaluation of the speckle detection using both simulated and real data. In addition, the applicability of the speckle information to signal to noise improvement in B-scan data will be investigated.

\section{Acknowledgments}

This work has been partially supported by the MEC grant nbs. TIN2005-08792-C03-01 and TIN2006-08035. Authors want to thank the Medical Imaging group at the Department of Engineering, University of Cambridge, UK, for the liver images used in this work.

\section{REFERENCES}

1. R. Prager, A. Gee, G. Treece, and L. Berman, "Speckle Detection in ultrasound images using first order statistics," Tech. Rep. TR 415, University of Cambridge, July 2001.

2. S. Ghofrani, M. Jahed-Motlagh, and A. Ayatollahi, "An adaptive speckle suppression filter based on Nakagami distribution," in IEEE EUROCON'2001, International Conference onTrends in Communications., 1, 2001.

3. B. I. Raju and M. A. Srinivasan, "Statistics of envelope of high-frequency ultrasonic backscatter from human skin in vivo," IEEE Trans Ultrasonics Ferroelectrics and Frequency Control 49(7), pp. 871-872, 2002.

4. M. Wachowiak, R. Smolikova, J. Zuranda, and A. Elmaghraby, "Estimation of K distribution parameters using neural networks," IEEE Transactions on Biomedical Engineering 49, pp. 617-620, June 2002.

5. R. Wagner, S. W. Smith, J. Sandrik, and H. Lopez, "Statistics of speckle in ultrasound B-scans," IEEE Transactions on Sonics and Ultrasonics 30, pp. 156-163, May 1983.

6. V. Dutt and J. Greenleaf, "Adaptative speckle reduction filter for Log-compressed B-scan images," IEEE Transactions on Medical Imaging 15, pp. 802-813, December 1996. 
7. X. Hao, C. Bruce, C.Pislaru, and J. Greenleaf, "Characterization of reperfused infarcted myocardium from high frequency intracardiac ultrasound imaging using Homodyned K distribution," IEEE Trans Ultrasonics Ferroelectrics and Frequency Control 49(11), pp. 1530-1542, 2002.

8. M. Martín-Fernández and C. Alberola-López, "On low order moments of the Homodyned-K distribution," Ultrasonics 43, pp. 283-290, 2005.

9. R. Momenan, R. F. Wagner, M. Loew, M. F. Insana, and B. S. Garra, "Characterization of tissue from ultrasound images," Control Systems Magazine, IEEE 8(3), pp. 49-53, 1988.

10. R. W. Prager, A. H. Gee, G. M. Treece, and L. H. Berman, "Analysis of speckle in ultrasound images using fractional order statistics and the Homodyned K-distribution," Ultrasonics 40, pp. 133-137, 2002.

11. V. Dutt, Statistical Analysis of Ultrasound Echo Envelope. PhD thesis, Mayo Graduate School, 1995.

12. R. Prager, A. Gee, G. Treece, and L. Berman, "Decompression and Speckle Detection For Ultrasound Images using the Homodyned k-Distribution," Pattern Recognition Letters 24, pp. 705-713, 2003.

13. R. Martí, J. Martí, J. Freixenet, J. Vilanova, and J. Barceló, "Optimally discriminant moments for speckle detection in real b-scan images," in Iberian Conference in Pattern Recognition (IbPRIA), Lecture Notes in Computer Science, (to be published), 2007.

14. A. Webb, Statistical Pattern Recognition, John Wiley and Sons, 2nd ed., 2003.

15. O. Michailovich and A. Tannenbaum, "Despeckling of medical ultrasound images," IEEE Transactions on Ultrasonics, Ferroelectrics, and Frequency Control 53(1), pp. 64-78, 2006.

16. R. J. Housden, A. H. Gee, G. M. Treece, and R. W. Prager, "Sensorless reconstruction of unconstrained freehand 3D ultrasound data," Tech. Rep. 533, University of Cambridge, Department of Engineering, May 2006.

17. J. Revell, M. Mirmehdi, and D. McNally, "Computer vision elastography: Speckle adaptive motion estimation for elastography using ultrasound sequences," IEEE Transactions on Medical Imaging 24(6), pp. 755$766,2005$. 